\title{
Time for an Alzheimer's disease cocktail
}

\author{
Warren Ladiges ${ }^{\mathrm{a}, *}$ \\ ${ }^{a}$ Department of Comparative Medicine, School of Medicine, University of Washington, Seattle, WA, USA.
}

\begin{abstract}
Treatment of Alzheimer's disease with drugs that target single mechanisms has not been successful. Therefore, new approaches must be considered. Since late onset AD is an age-related disease it shares many of the same mechanistic processes of growing old and should benefit from a multidrug targeting approach designed to enhance healthy aging. Aging intervention drugs are available to combine as cocktails for expanding the coverage from just one to multiple aging pathways. This geroscience concept is set to replace the narrow-focused neuroscience approach of trying to identify a single magic bullet target that has seen only failure. Scientific review groups and funding agencies must recognize the opportunity to fund preclinical and clinical research before Alzheimer's disease patients can benefit from a multitargeted anti-aging approach that is currently underappreciated both clinically and scientifically.
\end{abstract}

Keywords: Alzheimer's disease, aging, aging processes, drug cocktails, Alzheimer's disease intervention

Late onset Alzheimer's disease (AD) is a disease of the elderly, and accounts for greater than 95 percent of all AD cases. Therefore, it can be said that AD is an age-related disease. But what does this really mean? It means that mechanisms involved in aging are also implicated in AD and include such processes as inflammation, epigenetic changes, vascular deficits, oxidative stress, mitochondrial and autophagic impairment (Figure 1). The geroscience concept posits that $\mathrm{AD}$ is not a single mechanistic disease caused by faulty APP processing and hyperphosphorylated tau. Rather, it is a multifactorial disease tightly linked with mechanisms of aging. If it can be shown that an individual mechanism is altered by a cocktail of drugs targeting multiple aging processes, then by definition, that mechanism must be associated with aging and the early development of AD.

Research in the biology of aging has made exponential progress over the last twenty years, with first genetic approaches to aging intervention, and then pharmacological approaches using molecular targets identified from genetic studies. Investigations in aging research are now underway in several countries designed to explore strategies for

\footnotetext{
* Corresponding author: Warren Ladiges

Mailing address: Department of Comparative Medicine, School of Medicine, University of Washington, Seattle, WA 98195, USA.

Email: wladiges@uw.edu

Received: 13 March 2020 / Accepted: 18 March 2020
}

targeting multiple mechanistic pathways with drug cocktails, because aging is a complex multifactorial process. So why not consider AD as an aging condition and use a similar multidrug approach? This is a platform that has yet to be part of established AD research engrained in neuroscience approaches designed to unravel a singular neurological target for the one magic bullet that would successfully treat $\mathrm{AD}$. Rather, if $\mathrm{AD}$ is viewed as a systemic aging problem in the elderly, then pharmacological testing can begin using drug cocktails. Such an approach will require preclinical testing of drug combinations in various cellular and animal models that attack distinct mechanisms known to be involved in aging as well as AD.

Many neuroscience purists do not agree with this labeling of $\mathrm{AD}$, and in fact, consider that neurological dysfunction is unrelated to systemic organ disease relative to the development and progression of AD neuropathology. No wonder attempts to treat and prevent AD have consistently resulted in failure. Neuroscience review groups that routinely reject grant applications that focus on aging as a driving factor in AD are denying opportunities to extend $\mathrm{AD}$ research into aging research and subsequently Alzheimer's disease patients. Aging mechanisms are conserved pathways, so drug cocktail studies are relevant in aging animal models challenged with $\mathrm{AD}$ and have already been started. Many questions are being addressed, such as type of drug, dosage, timing, drug interaction, prevention or treatment.

Clinical trials are needed to establish efficacy and safety of drug cocktails vital to successfully treat AD in people. Just as importantly, decisions will have to be made on 


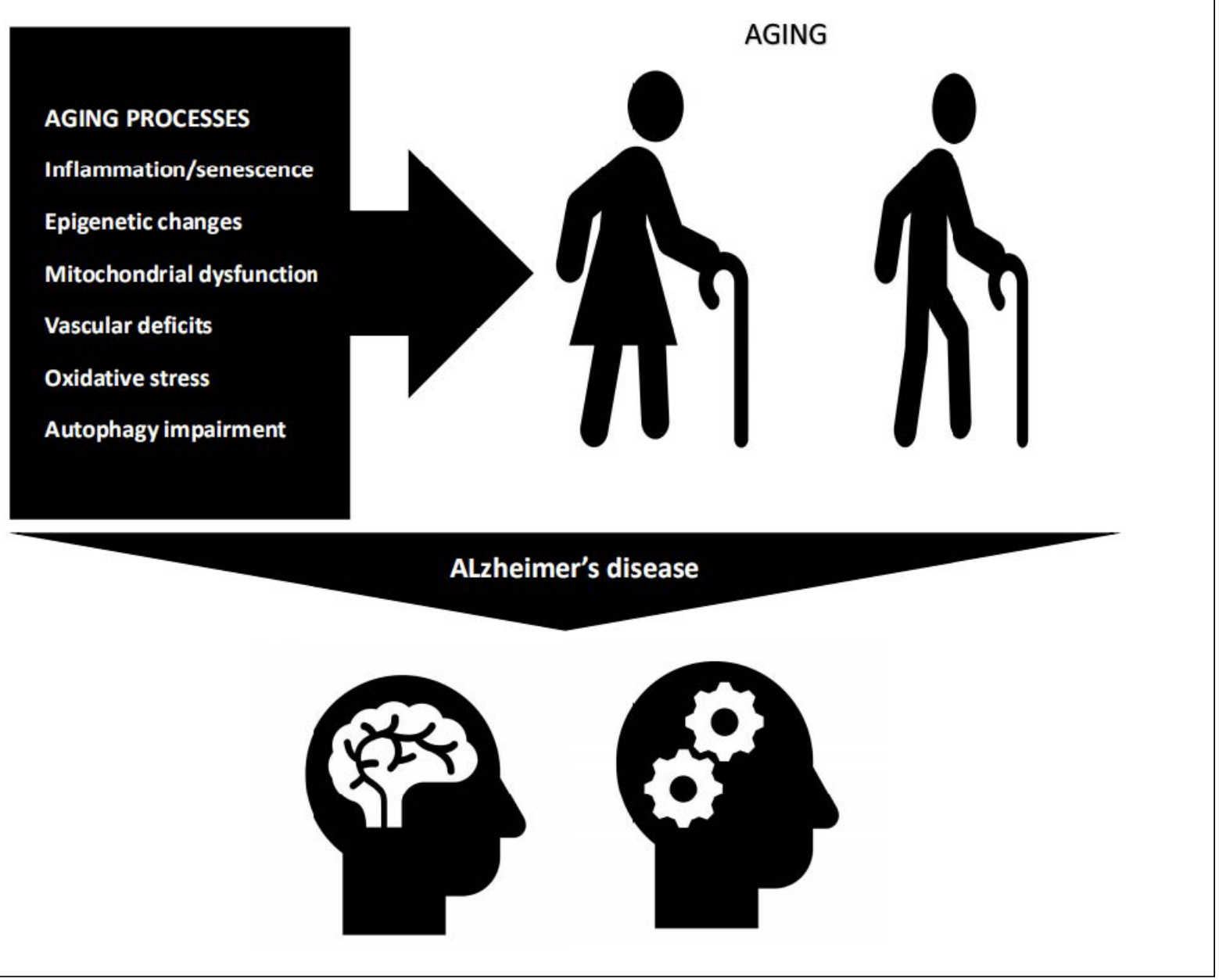

Figure 1. Alzheimer's disease is a complex pathological condition associated with multiple processes of aging including inflammation/senescence, epigenetic changes, mitochondrial dysfunction, vascular deficits, oxidative stress, and autophagy impairment. These processes can be targeted more effectively with drug cocktails rather than with an individual drug.

whether the emphasis should be on trying to treat advanced $\mathrm{AD}$ to help enhance quality of life or treating middle age people with or without mild cognitive impairment to prevent the progression of neuropathology suspected to be driven by AD. Since drug cocktails are designed to target aging processes, early treatment rather than late treatment would likely be more successful. Genetic and clinical diagnostic testing could identify persons at risk. Is it clinically okay to treat asymptomatic people with an anti-aging drug cocktail with the purpose of slowing aging and preventing the progression of early neuropathological lesions? One of the strategies in pharmaceutical aging research is testing intermittent or short-term administration of drug cocktails in animal model studies and monitor for residual effects after treatment. Another strategy is to use stress tests for determining those with low physical resilience to aging and $\mathrm{AD}$ as a way to select candidates for pretreatment with a drug cocktail.

These are issues that can be addressed with clinical trials, but there is an urgent need to start now with initial studies using drug cocktails shown to be effective in preventing mild cognitive impairment and early brain lesions associated with development of AD in animal models. Funding for moving this strategy forward is critical. Successful treatment and prevention, or even a delay in the onset of $\mathrm{AD}$, would have a tremendous impact on the health of patients and their families and save millions of dollars in health care costs.

Cite this article as: Ladiges W C. Time for an Alzheimer's disease cocktail[J]. Aging Pathobiology and Therapeutics, 2020, 2(1): 14-15. 